\title{
Laser-Induced Microexplosion Confined in the Bulk of a Sapphire Crystal: Evidence of Multimegabar Pressures
}

\author{
S. Juodkazis, ${ }^{1}$ K. Nishimura, ${ }^{1}$ S. Tanaka, ${ }^{1}$ H. Misawa, ${ }^{1}$ E. G. Gamaly, ${ }^{2}$ B. Luther-Davies,${ }^{2}$ \\ L. Hallo, ${ }^{3}$ P. Nicolai, ${ }^{3}$ and V. T. Tikhonchuk ${ }^{3}$ \\ ${ }^{1}$ CREST-JST and Research Institute for Electronic Science, Hokkaido University, N21-W10, \\ CRIS Building, Kita-ku, Sapporo 001-0021, Japan \\ ${ }^{2}$ Centre for Ultrahigh Bandwidth Devices for Optical Systems, Laser Physics Centre, \\ Research School of Physical Sciences and Engineering, The Australian National University, Canberra ACT 0200, Australia \\ ${ }^{3}$ Centre Lasers Intenses et Applications, UMR 5107 CEA CNRS - Université Bordeaux 1, 33405 Talence, Cedex, France
}

(Received 24 November 2005; published 25 April 2006)

\begin{abstract}
Extremely high pressures $(\sim 10 \mathrm{TPa})$ and temperatures $\left(5 \times 10^{5} \mathrm{~K}\right)$ have been produced using a single laser pulse $(100 \mathrm{~nJ}, 800 \mathrm{~nm}, 200 \mathrm{fs})$ focused inside a sapphire crystal. The laser pulse creates an intensity over $10^{14} \mathrm{~W} / \mathrm{cm}^{2}$ converting material within the absorbing volume of $\sim 0.2 \mu \mathrm{m}^{3}$ into plasma in a few fs. A pressure of $\sim 10 \mathrm{TPa}$, far exceeding the strength of any material, is created generating strong shock and rarefaction waves. This results in the formation of a nanovoid surrounded by a shell of shock-affected material inside undamaged crystal. Analysis of the size of the void and the shock-affected zone versus the deposited energy shows that the experimental results can be understood on the basis of conservation laws and be modeled by plasma hydrodynamics. Matter subjected to record heating and cooling rates of $10^{18} \mathrm{~K} / \mathrm{s}$ can, thus, be studied in a well-controlled laboratory environment.
\end{abstract}

DOI: 10.1103/PhysRevLett.96.166101

The study of matter in conditions of extreme pressure and temperature is an exciting area of condensed matter physics relevant to the formation of new materials and modeling the state of matter inside stars and planets. Creation of such extreme conditions in the laboratory is, however, a formidable experimental task. So far, pressures in excess of $0.1 \mathrm{TPa}$ have been obtained using a diamond anvil in stationary conditions, while transient pressures behind shock waves generated by chemical or nuclear explosions or generated using powerful lasers up to $50 \mathrm{TPa}$ have been reported [1,2]. Here we present experimental evidence that one can create TPa pressures, many times the strength of any material, using low energy pulses from a conventional tabletop laser.

Recent studies have demonstrated [3-8] that sub-ps laser pulses tightly focused inside transparent dielectrics (glasses, crystals, and polymers) can produce detectable sub-micrometer-sized structural modifications, including voids. This requires intensities in excess of $10^{14} \mathrm{~W} / \mathrm{cm}^{2}$ which results in a highly nonlinear light-matter interaction with most dielectrics being ionized early in the laser pulse. To achieve such high intensities the laser beam should be tightly focused using high numerical aperture optics into a spot whose dimensions are of the order of the laser wavelength $(\lambda)$.

Previously the formation of voids in silica was associated with self-focusing of the laser beam [3]. In fact void formation can occur in conditions favorable for selffocusing if the beam is weakly focused into the sample [9]. Previously, however, there has been no systematic study of void formation by single fs pulses in conditions when self-focusing cannot occur. We demonstrate here that in such conditions nanovoids are formed by the extreme
PACS numbers: 81.07. $-\mathrm{b}, 47.40 . \mathrm{Nm}, 62.50 .+\mathrm{p}, 81.40 .-\mathrm{z}$

temperatures and pressures created by optical breakdown and these drive shock and rarefaction waves in the surrounding material. It is possible that new materials [10-12] with altered chemical properties [13] could be formed by such micro-explosions.

The interaction between an intense laser pulse and the material is fundamentally different when the laser beam is tightly focused inside a transparent solid rather than on its surface. Inside the solid the interaction zone containing high-energy density is confined inside cold and dense material. For this reason hydrodynamic expansion is either insignificant or highly restricted. In our experiments $100 \mathrm{~nJ}, 150 \mathrm{fs}$, laser pulses at $800 \mathrm{~nm}$ were tightly focused using high numerical aperture optics ( $N A \equiv n \sin \theta=1.35$ where $\theta \simeq 50^{\circ}$ is the beam convergence angle) inside a sapphire crystal (the sapphire band gap is $\Delta_{g}=8.8 \mathrm{eV}$ ) into a focal volume less than $\lambda^{3}$.

To avoid self-focusing, and thus deliver the energy in the laser pulse to a specific location inside the transparent solid, requires the laser power to be lower than the critical value for self-focusing. This critical power depends on the nonlinear part of the refractive index $n_{2}\left(n=n_{0}+n_{2} I\right)$ as follows [14], $P_{\mathrm{cr}}=\lambda^{2} / 2 \pi n_{0} n_{2}$. The critical power of $1.7 \mathrm{MW}$ for sapphire $\left[n_{2}=3.54 \times 10^{-16} \mathrm{~cm}^{2} / \mathrm{W}\right.$ [15] ] is much higher than the peak power of $0.5 \mathrm{MW}$ used in our experiments. Therefore, the laser energy could be delivered to the focal volume located from 5 up to $50 \mu \mathrm{m}$ below the crystal surface without distortion.

An array of laser-affected spots was created aligned along the $c$ plane inside the sapphire crystal using a sequence of single laser pulses. The pulse energy was in a range $20-120 \mathrm{~nJ}$ and even the lowest intensity used significantly exceeded the optical breakdown threshold. The 
sample was then cleaved along the $c$ plane and examined using a scanning electron microscope (SEM). The observed pattern is shown in Fig. 1. Careful examination revealed that the region irradiated by a single laser pulse consisted typically of a central void surrounded by a shell extending to about twice the void diameter. This shell was identified as amorphous material by chemical etching since amorphous sapphire has a much higher solubility in hydrofluoric acid compared with crystalline sapphire. Indeed, the shell could be etched away completely using a $10 \%$ aqueous solution of HF up to a smooth boundary with the pristine sapphire crystal (Fig. 2). Thus, the exact size of shock-wave-affected amorphous zone (as discussed below) could be established. One can see from Fig. 2 that the lasermodified regions are teardrop-shaped along the direction of pulse propagation due to spherical aberration caused by the refractive index mismatch between the immersion oil $(n=$ $1.515)$ and sapphire $(n=1.757)$.

We define the focal volume as that confined inside the cylindrical surface where the intensity equals $1 / 2$ of its maximum value. The radius of this cylinder for a Gaussian beam is $r_{1 / 2} \simeq \sqrt{\ln 2 / 2} r_{0}$ [16]. The radius of the first Airy disk for a plane wave focusing approximately equals the radius of the Gaussian beam where the intensity drops to $1 / e^{2}$ of the maximum value and can be estimated as $r_{0} \equiv$ $0.61 \lambda /$ NA. The axial length of the focal volume is twice the Rayleigh length $2 z_{0}=2 \pi r_{0}^{2} n / \lambda$. Hence, the focal volume $V_{1 / 2}=0.947 \lambda^{3} n / \mathrm{NA}^{4}$, and focal spot area $S_{1 / 2}=$ $0.405 \lambda^{2} / \mathrm{NA}^{2}$. For our experiments the focal volume $V_{1 / 2}=0.288 \mu \mathrm{m}^{3}$ and the focal area was $S_{1 / 2}=$ $0.153 \mu \mathrm{m}^{2}$ leading to an intensity of $3.5 \times 10^{14} \mathrm{~W} / \mathrm{cm}^{2}$ for a $100 \mathrm{~nJ}$ pulse.

It is well established [17-25] that multiphoton ionization [26] and the ionization by electron impact are responsible for the optical breakdown in dielectrics. In our case a minimum six photons is required, $n_{\mathrm{ph}}=\Delta_{g} / \hbar \omega \sim 6$, to transfer an electron from the valence to the conduction band. The six photon ionization rate $[18,26] w_{\mathrm{mpi}} \simeq$ $\omega n_{\mathrm{ph}}^{3 / 2}\left(\varepsilon_{\mathrm{osc}} / 2 \Delta_{g}\right)^{n_{\mathrm{ph}}} \sim 3.5 \times 10^{16} \mathrm{~s}^{-1}$ is a rather fast pro-

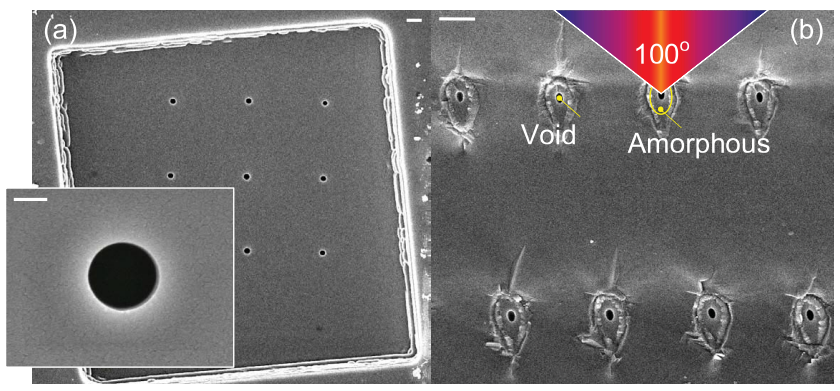

FIG. 1 (color). Top (a) and side-view (b) SEM images of a pattern of voids produced by single $150 \mathrm{fs}, 800 \mathrm{~nm}$ wavelength, $120 \mathrm{~nJ}$ (at focus) pulses in sapphire. A focused ion beam mill was used to generate the transverse cross section of voids located $5 \mu \mathrm{m}$ beneath the surface in (a), while the as-cleaved side plane is shown in (b). The cone angle of the focused beam, $2 \theta$, is also shown (b). Scale bars: $1 \mu \mathrm{m}$; $0.1 \mu \mathrm{m}$ [inset in (a)]. cess (here, $\varepsilon_{\text {osc }}$ is the quiver energy of electrons in the laser field). The avalanche ionization model for dielectrics has been recently revisited and improved [24,25]. The ionization rate in a dielectric with a band gap $\Delta_{g}$ can be expressed through the effective electron collision frequency [18]: $w_{a} \simeq\left(\varepsilon_{\mathrm{osc}} / \Delta_{g}\right) \omega^{2} \nu_{\mathrm{eff}} /\left(\nu_{\mathrm{eff}}^{2}+\omega^{2}\right)$. The process of energy absorption by oscillating electrons occurs due to electron-phonon collisions early in the pulse, $\nu_{\text {eff }} \approx$ $\nu_{e-\text { ph }} \ll \omega$, and later by electron-ion collisions $\nu_{\text {eff }} \simeq$ $\nu_{e-i} \sim \omega_{\mathrm{pe}}>\omega$, where $\omega_{\mathrm{pe}}$ is the electron plasma frequency. The avalanche ionization rate in both limiting cases during the laser pulse $\left(\omega \gg \nu_{e-\mathrm{ph}} \sim 10^{14} \mathrm{~s}^{-1} ; \omega \ll\right.$ $\nu_{e-i} \sim \omega_{\mathrm{pe}} \sim 1.5 \times 10^{16} \mathrm{~s}^{-1}$ ) is approximately $w_{a} \simeq$ $(0.3-1) \times 10^{15} \mathrm{~s}^{-1}$. The interplay of two processes, multiphoton and avalanche ionization, swiftly converts the irradiated part of crystal into solid density plasma (electron density $n_{e} \sim 10^{23} \mathrm{~cm}^{-3}$; average ion charge of 4-5) in only a few fs early in the pulse.

In a solid density plasma recombination proceeds mainly by three-body collisions with one electron acting as the third body [27]. The characteristic recombination time in the conditions of the experiments is $t_{r} \sim 30 \mathrm{fs}$. Thus, local ionization equilibrium is established by the end of the laser pulse. Since the electron density exceeds the critical density for the laser frequency, the absorption length and the absorption volume both collapse due to transformation into the plasma state resulting in a sharp increase in the energy density deposited by laser.

In a solid density plasma at $n_{e} \sim 10^{23} \mathrm{~cm}^{-3}$, the electron-ion collision frequency is of the order of the plasma frequency, $\nu_{e i} \sim \omega_{\mathrm{pe}} \simeq 2 \times 10^{16} \mathrm{~s}^{-1}$ [28]. Thus the imaginary part of the dielectric function equals $\epsilon^{\prime \prime} \approx$ $\omega_{\mathrm{pe}} / \omega \sim 7.59$, and the real and imaginary parts of the refractive index are the same, $n \approx k=\sqrt{\epsilon^{\prime \prime} / 2} \simeq 2$. The absorption length then shrinks, $l_{s}=c / \omega k=65 \mathrm{~nm}$, while the absorption coefficient increases $A \simeq 4 n /\left[(n+1)^{2}+\right.$ $\left.k^{2}\right] \simeq 0.61$. Thus, the laser energy becomes efficiently absorbed in a volume significantly smaller than the focal volume, $V_{\mathrm{abs}}=l_{s} \pi r_{1 / 2}^{2}=10^{-2} \mu \mathrm{m}^{3}$.

The electron-ion energy exchange rate in solid density plasma is $\nu_{T} \sim\left(m_{e} / M\right) \nu_{\text {eff }}=4.78 \times 10^{11} \mathrm{~s}^{-1}$ [29]. There-

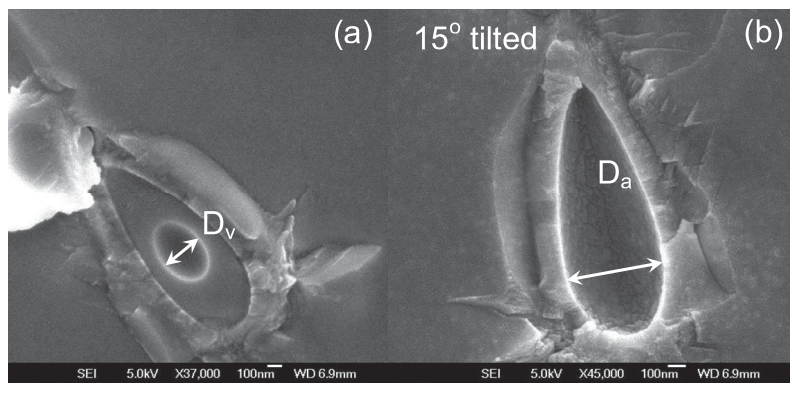

FIG. 2. SEM images of a laser-affected region produced by the $150 \mathrm{fs}, 800 \mathrm{~nm}, 100 \mathrm{~nJ}$ pulses in sapphire $15 \mu \mathrm{m}$ beneath the sapphire surface before (a) and after (b) etching for $5 \mathrm{~min}$ in $10 \%$ aqueous solution of hydrofluoric acid. 
fore, the energy equilibration time, $2 \mathrm{ps}$, is much longer than the pulse duration. The energy deposited by the end of the pulse results in electron heating while the ions remain cold. The electron energy density in the absorbing region at the end of the pulse can be estimated taking ionization losses into account as $W_{\text {dep }}=2 A F_{p} / l_{s}-E_{\text {ion }}$, where $F_{p}$ is the pulse fluence, and the second term includes the total losses to ionization for aluminum and oxygen to charge states $Z_{\mathrm{Al}}$ and $Z_{\mathrm{O}}$. However, because the recombination time is shorter than the pulse duration, ionization equilibrium is established by the end of the pulse and the electron density, therefore, saturates. Thus, the maximum pressure which subsequently drives a shock wave can be simply estimated from the total deposited energy $\left(E_{p}=100 \mathrm{~nJ}\right.$, $\left.F_{p}=70.4 \mathrm{~J} / \mathrm{cm}^{2} ; S_{1 / 2}=0.15 \mu \mathrm{m}^{2}, l_{s}=65 \mathrm{~nm} ; A=0.61\right)$ and the absorbing volume, $p_{\max } \simeq 13.4 \mathrm{TPa}$ ), and this significantly exceeds the Young's modulus of sapphire, $Y=$ $0.4 \mathrm{TPa}$.

Experimentally the dependence of the diameter of the laser-affected region (the boundary between amorphous and crystal sapphire) was measured for increasing pulse energy and the same focusing conditions [Fig. 3(a)]. The central void only appeared when the pulse energy was greater than 20-25 nJ. At this threshold the laser-affected region extended to $\sim 250 \mathrm{~nm}$, but the central void was absent. Examination of the laser-affected region created in these conditions revealed that this area had lower density than the surrounding crystal as revealed by differences in charging during SEM imaging. The pressure driving the shock wave at this energy already exceeded the Young modulus by 3-4 times; hence, it created an amorphous region but was not strong enough to create a void. No cracks were observed in the crystal surrounding the laseraffected zone for pulse energies in the range 20-100 nJ. The onset of crack formation occurred at pulse energies above $100 \mathrm{~nJ}$ when the diameter of the amorphous region exceeded $500 \mathrm{~nm}$.

The formation of a void and shock-affected zone can be understood from the laws of mass and energy conservation. Consider for simplicity spherically symmetric motion. The shock wave propagating in a cold material loses its energy
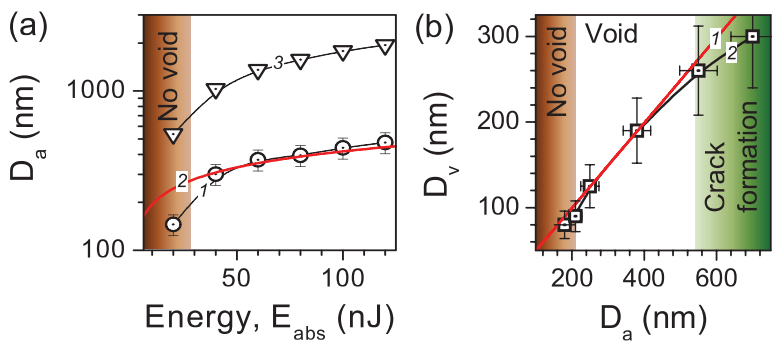

FIG. 3 (color). (a) The diameter (1) and length (3) of the amorphous region vs the absorbed pulse energy, $E_{\mathrm{abs}}$. The voids were $20 \mu \mathrm{m}$ beneath the surface. Curve (2) plotted by Eq. (1) with $l_{a}=80 \mathrm{~nm}$. (b) Dependence of the void diameter on the diameter of amorphous part: (1) theory by Eq. (2) with $\delta=1.14$; (2) experiment. due to dissipation due to the work done against the internal pressure (Young modulus) that resists material compression. The distance at which the shock front effectively stops defines the shock-affected volume. At this point the shock wave converts into a sound wave, which propagates further into the material without inducing any permanent changes. The distance where the shock wave stops can be estimated from the condition that the internal energy in the volume inside the shock front is comparable to the absorbed energy: $\frac{4}{3} \pi r_{s}^{3} p_{0}=E_{\text {abs. }}$. In other words, at this position the pressure behind the shock front equals the internal pressure of the cold material [27]. One can reasonably suggest that the sharp boundary observed between the amorphous (laser-affected) and crystalline (pristine) sapphire corresponds to the distance where the shock wave effectively stopped. The experimentally measured dependence of the laser-affected zone diameter, $D_{a}$, (outer size of amorphous region) on the laser energy can be fitted according to the argument above by following relationship

$$
D_{a}=l_{a} \sqrt[3]{E_{\mathrm{abs}}}
$$

where $l_{a}=80 \mathrm{~nm}$ and $E_{\mathrm{abs}}$ is in nJ. This value of the characteristic length, $l_{a}$, at the threshold energy is in fact close the absorption depth for plasma, $l_{s}$, as calculated above.

Similarly one can apply the mass conservation law to estimate the density of compressed material from the void size. Void formation inside a solid is only possible if the mass initially contained in the volume of the void was pushed out and compressed. Thus after the microexplosion the whole mass initially confined in a volume with diameter $D_{a}$ resides in a spherical layer in between $D_{a}$ and $D_{v}$, which has a density $\rho=\rho_{0} \delta$ with $\delta>1$. The void diameter can be expressed through the compression ratio and the diameter of laser-affected zone with the help of mass conservation as follows:

$$
D_{v}=D_{a} \sqrt[3]{1-1 / \delta}
$$

Typically we observed $D_{v} \sim 0.5 D_{a}$ which means that amorphous material has a density 1.14 times higher than that of crystalline sapphire.

Computer simulations were performed in spherical geometry using the hydrodynamic code Chivas [30] (ionization equilibrium, separate temperatures of electron and ions, electron heat conduction). We approximated the cylindrical region where the energy was absorbed by an equivalent sphere, $V_{\mathrm{abs}}=\pi r_{1 / 2}^{2} l_{s}=\frac{4}{3} \pi r_{\mathrm{dep}}^{3}$. The energy $E_{\text {abs }}=50 \mathrm{~nJ}$ was deposited homogeneously in this spherical volume with radius $r_{\text {dep }}=0.13 \mu \mathrm{m}$ in a material with initial density $\rho_{0}$. The equation of state (EOS) implemented in the code [31] describes solid-melt-plasma and reverse transitions. The simulations started after the end of the laser pulse with the initial electron temperature $T_{e}=$ $26.2 \mathrm{eV}$ and the average ion charge (number of electrons that are stripped off an atom) $\langle Z\rangle=4.3$. The hydrodynamic motion commences after the electrons transfer the 

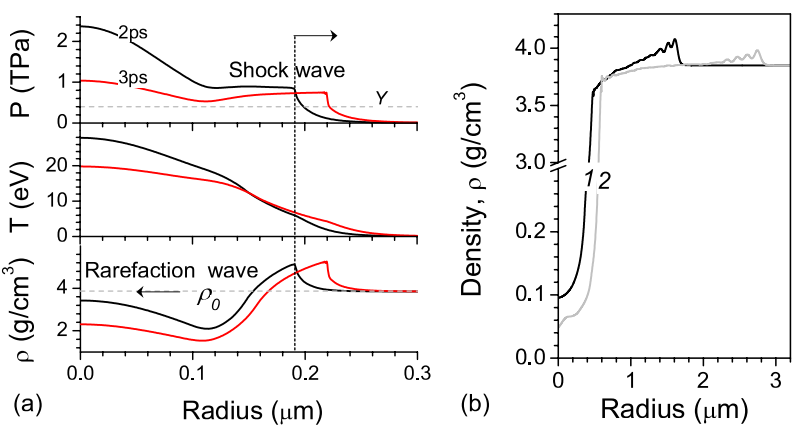

FIG. 4 (color online). (a) Spatial profiles of pressure, $P$, temperature, $T$, and mass density, $\rho$, at 2 and 3 ps after the laserinduced micro-explosion in sapphire. (b) Density profiles at 0.1 (1), $0.2 \mathrm{~ns}(2)$ for a $50 \mathrm{~nJ}$ deposited inside a $0.13 \mu \mathrm{m}$ radius volume. The void has already formed and a weak shock wave propagates outside it with velocity $\sim 11.46 \mathrm{~km} / \mathrm{s}$ (sound velocity is $10 \mathrm{~km} / \mathrm{s}$ ). The density $\rho_{0}=3.89 \mathrm{~g} / \mathrm{cm}^{3}$ and Young modulus $Y=400 \mathrm{GPa}$ of sapphire are shown by the dashed lines in (a).

absorbed energy to ions in excess of that necessary to break interatomic bonds. The strong shock wave emerges at the outer surface of sphere where the energy was deposited compressing the material to a density twice its initial value. Subsequently, the pressure behind the shock front rapidly decreases with distance, and finally the shock transforms into an acoustic wave. A void is formed behind the shock front and its radius gradually increases. The spatial density profiles for the time instants 0.1 and 0.2 ns are shown in Fig. 4(b). The compression ratio at $1 \mathrm{~ns}$ reaches its asymptotic value of $\delta=\rho / \rho_{0}=1.1$ which qualitatively agrees with the density of the amorphous layer deduced from the experiments, $\delta_{\exp }=1.14$. We note that the remaining gas density in the central void region at $1 \mathrm{~ns}$ is above $0.02 \mathrm{~g} / \mathrm{cm}^{3}$. These hydrodynamic calculations predict the state of the laser-affected solid for the period up to $1 \mathrm{~ns}$ after the pulse in reasonable agreement with the experimental data.

The micro-explosion in sapphire is characterized by the total absorbed energy $E_{0}$, initial density $\rho_{0}$, and radius of the energy deposition zone, $R_{0}$, because the energy deposition time (picoseconds) is negligible compared with the characteristic hydrodynamic time (nanoseconds). As a result, the hydrodynamic equations can be reduced to the set of ordinary equations with one variable [27], $\xi=r / c_{0} t$, where $c_{0} \sim \sqrt{p_{0} / \rho_{0}}$ is the characteristic acoustic velocity, describing any hydrodynamic phenomena with the same initial pressure and density. A wide variety of explosive phenomena are thus only distinguished by their different space, $R_{0}$, and time scales $t_{0}=\sqrt{r_{\mathrm{dep}}^{5} \rho_{0} / E_{\mathrm{abs}}}$. The similarity laws of hydrodynamics thus suggest that a microexplosion in sapphire $\left(E_{0}=0.1 \mu \mathrm{J}, \rho_{0} \simeq 4 \mathrm{~g} / \mathrm{cm}^{3}, R_{0}=\right.$ $150 \mathrm{~nm}, t_{0}=5.5 \mathrm{ps}$ ) is simply a reduced copy of a macroscopic explosion that produces the same pressure at the same initial density but with the distance and time scales changed in accordance with the above formulas. Hence, experiments using energy densities normally available only at the high-energy laser facilities dedicated to studies of inertial confinement fusion [2] can apparently be reproduced in laboratory tabletop experiments.

To conclude, we have generated and analyzed the unique conditions that can be created by a single pulse from a tabletop fs laser focused inside crystalline sapphire. Extreme pressures of $10 \mathrm{TPa}$, temperatures of $5 \times 10^{5} \mathrm{~K}$, and record high heating and cooling rates of $10^{18} \mathrm{~K} / \mathrm{s}$ were generated. This provides the exciting possibility to study matter in extreme conditions in a well-controlled laboratory environment.

[1] P. F. McMillan, Nat. Mater. 4, 715 (2005).

[2] J. D. Lindl et al., Phys. Plasmas 11, 339 (2004).

[3] E. N. Glezer et al., Opt. Lett. 21, 2023 (1996).

[4] C. B. Schaffer et al., Opt. Lett. 26, 93 (2001).

[5] K. Yamasaki et al., Appl. Phys. Lett. 76, 1000 (2000).

[6] H. Sun et al., Opt. Lett. 26, 325 (2001).

[7] C. Hnatovsky et al., Appl. Phys. Lett. 87, 014104 (2005).

[8] D. Day and M. Gu, Appl. Phys. Lett. 80, 2404 (2002).

[9] A. Dubietis et al., Phys. Rev. Lett. 92, 253903 (2004).

[10] Q. Johnson and A.C. Mitchell, Phys. Rev. Lett. 29, 1369 (1972).

[11] A. B. Belonoshko, Science 275, 955 (1997).

[12] S.-D. Mo and W. Y. Ching, Phys. Rev. B 57, 15219 (1998).

[13] G. E. Duvall et al., Nature (London) 296, 846 (1982).

[14] S. Akhmanov, V. Vyspoukh, and A. Chirkin, Optics of Femtosecond Laser Pulses (Nauka, Moscow, 1988).

[15] Handbook of Optics: Devices, Measurements, \& Properties, edited by M. Bass (McGraw-Hill, New York, 1995), 2nd ed., Vol. II.

[16] M. Born and E. Wolf, Principles of Optics (Cambridge University Press, Cambridge, England, 2003), 7th ed.

[17] E. Yablonovitch and N. Bloembergen, Phys. Rev. Lett. 29, 907 (1972).

[18] Y.P. Raizer, Laser-Induced Discharge Phenomena (Consultants Bureau, New York, 1977).

[19] D. Arnold and E. Cartier, Phys. Rev. B 46, 15102 (1992).

[20] D. von der Linde and H. Schuler, J. Opt. Soc. Am. B 13, 216 (1996).

[21] B. Stuart et al., Phys. Rev. Lett. 74, 2248 (1995).

[22] A.-C. Tien et al., Phys. Rev. Lett. 82, 3883 (1999).

[23] C. W. Carr et al., Phys. Rev. Lett. 92, 087401 (2004).

[24] B. Rethfeld, Phys. Rev. Lett. 92, 187401 (2004).

[25] B. Rethfeld, Phys. Rev. B 73, 035101 (2006).

[26] L. V. Keldysh, Sov. Phys. JETP 20, 1307 (1965).

[27] Y. B. Zel'dovich and Y.P. Raizer, Physics of Shock Waves and High-Temperature Hydrodynamic Phenomena (Dover, New York, 2002).

[28] E. G. Gamaly et al., Phys. Plasmas 9, 949 (2002).

[29] W.L. Kruer, Physics of Laser Plasma Interactions (Addison-Wesley, Reading, MA, 1988).

[30] S. Jacquemot and A. Decoster, Laser Part. Beams 9, 517 (1991).

[31] R. More et al., Phys. Fluids 31, 3059 (1988). 